\title{
The Diffuse Extreme Ultraviolet Background
}

\author{
John Vallerga and Jonathan Slavin \\ Eureka Scientific Inc., 2452 Delmer St. Suite 100, Oakland, CA 94602-3017 USA
}

\begin{abstract}
Observations of the diffuse EUV background towards 138 different directions using the spectrometers aboard the Extreme Ultraviolet Explorer satellite (EUVE) have been combined into a spectrum from $150 \AA$ to $730 \AA$ and represent an effective exposure of 18 million seconds. There is no significant evidence of any non-local line flux in the resultant spectrum such as that from a hot coronal plasma. These results are inconsistent with the Wisconsin $\mathrm{C}$ and $\mathrm{B}$ broad-band surveys assuming the source is a $\log T=5.8-6.1$ hot plasma in ionization equilibrium with solar abundances, confirming the previous result of Jelinksy, Vallerga \& Edelstein (1995) (hereafter Paper I) using an observation along the ecliptic with the same instrument. To make these results consistent with the previous broad-band surveys, the plasma responsible for the emission must either be depleted in $\mathrm{Fe}$ by a factor of $\sim 6$, be behind an absorbing slab of neutral $\mathrm{H}$ with a column of $2 \times 10^{19} \mathrm{~cm}^{-2}$, or not be in collisional ionization equilibrium (CIE). One such non-CIE model (Breitswerdt \& Schmutzler, 1994) that explains the soft $\mathrm{x}$-ray results is also inconsistent with this EUV data.
\end{abstract}

\section{Why Investigate the Diffuse EUV Background?}

If the local bubble is filled with a solar abundance hot plasma $(\log T=6)$ in equilibrium, then its dominant cooling mechanism is line emission in the EUV, specifically the Fe line complex near $170-190 \AA(72-64 \mathrm{eV})$ If detected, this emission must be reasonably local, as one optical depth at $170 \AA$ corresponds to a neutral $\mathrm{H}$ column of $10^{19} \mathrm{~cm}^{-2}$. Some non-CIE models of the Local Bubble, such as the adiabatic expansion of very hot ionized plasma (Breitswerdt \& Schmutzler, 1994) can reproduce similar soft $x$-ray broad band count rates but differ markedly with CIE models in the EUV, since the effective temperature of the electrons is only $40,000 \mathrm{~K}$. The EUV flux is therefore a strong discriminant between CIE and non-CIE models, Fe abundances in the hot gas and local absorption effects.

Though not designed as an diffuse instrument, the spectrometers aboard EUVE have a small but finite area-solid angle product ( $A \Omega=.00005 \mathrm{~cm}^{-2} \mathrm{SR}$ ), especially at the wavelengths of the Fe VIII-X complex near $170 \AA$ where the emission from a million degree plasma is the strongest. The spectometers observe this background in parallel with the point source obserations of EUVE Guest Observers, resulting in a combined data set of 18 million seconds of effective exposure during the first two years of the EUVE mission. 


\section{Data Reduction, Results and Discussion}

Each of the 248 pointings of the EUVE spectrometers were reduced identically. The technique for converting the two dimensional spectral image into a one dimensional count rate spectrum with errors can be found in Paper 1. A subsection of the combined and fluxed spectrum of 18 million seconds exposure can be seen in Fig. 1. We have concentrated our attention on this spectral region as it is far from the $\mathrm{He} \mathrm{I}$ and HeII geocoronal lines, is less affected by intestellar absorption than the longer wavelengths, and is most sensitive to $a \log T \sim 6$ plasma.

The EUVE Medium $(140 \AA-380 \AA)$ and Long $(280 \AA-730 \AA)$ wavelength spectrometers both have a wire grid colimator to spectrally limit the geocoronal background flux, thus providing a triangular spectral signature to any diffuse monochromatic radiation. Intrinsic detector background was removed from this spectrum by determining the background under a filter support structure that shades the detector. A residual smooth continuous background has been modeled with a quadratic polynomial funtion of wavelength. Spectral models of the diffuse background were then convolved through the collinator and telescope/detector response functions, added to the quadratic background and compared to the one dimensional count rate spectra.

To model the diffuse EUV flux from the Local Bubble, we have used a chisquared fitting procedure using Raymond and Smith (R\&S) thermal plasma code parameterized by temperature and emission measure (EM) as well as a single case of a Breitschwerdt \& Schmultzler (B\&S) non-CIE model where $\log \mathrm{T}=4.6, \log \mathrm{n}_{e}=-1.6$ and the flux is linearly parameterized by distance through the emitting gas. In all cases we have assumed a foreground slab of neutral hydrogen with a column of $10^{18} \mathrm{~cm}^{-2}$. We also let the three quadratic background parameters vary in each fit. Fig. 2 shows the predicted EUV spectra of each model with parameters chosen to fit the average Wisconsin $B$ band flux: EM of $.0036, \log T=6.0$ for the R\&S model and $100 \mathrm{pc}$ for the $\mathrm{B} \& \mathrm{~S}$ model. Contrasting with the strong $\mathrm{Fe}$ emission lines of the CIE plasma codes, the B\&S model is dominated by the Hell recombination continuum emission shortward of the edge at $228 \AA$. Figure 3 shows these models convolved through the instrument, as well as the residual of the measured spectrum after a simple quadratic fit has been subtracted. Though the instrumental resolution is poor, it is evident that the parameters that fit the xray results strongly overpredict the EUV flux. The formal fit to the EUVE data give an $\mathrm{EM}$ of $.00057 \pm .00014 \mathrm{~cm}^{-6} \mathrm{pc}$ for $\log \mathrm{T}=6.0$ and a pathlength of $21 \pm 4.6 \mathrm{pc}$ for the $\mathrm{B} \& \mathrm{~S}$ model. Rather than claiming a marginal detection at this level, which requires a detailed understanding and quantization of our sytematic errors, we quote a $2 \sigma$ upper limit of $\mathrm{EM}=.00085 \mathrm{~cm}^{-6} \mathrm{pc}$ or a maximum pathlength of $30 \mathrm{pc}$.

When the 138 directions on the sky are analysed separately and fit with a $\log T=6.0 \mathrm{R} \& \mathrm{~S}$ model, none of the observations detect an EM greater than a 3 sigma confidence limit. However, there is a hint that the data favor 
flux from Fe lines rather than HeII recombination. If we separate the 138 directions by their $B$ count rate, those above $75 \mathrm{cps}$ have a weighted mean $\mathrm{EM}$ of $.0011 \pm .00032$ while those directions with a count rate below $75 \mathrm{cps}$ have a weighted mean of $.00036 \pm .00016$, a significant difference indicating a correllation with the $\mathrm{B}$ band. Also, the four observations with the highest positive significance are in directions of a high $\mathrm{B}$ band count rate. The derived EMs do not seem to correlate with low column directions such as towards the galactic poles or towards the Canis Majoris direction in the galactic third quadrant. For the non-CIE model of $B \& S$, there was no correlation of the results with $B$ band count rates.

The thermal plasma models that are used to explain the soft xray rocket and satellite data predict EUV flux assuming low absorbing column, solar abundances and equilibrium conditions and all three could be wrong. We chose to assume a low foreground column based on the knowledge that except in a few directions toward the galactic center, the neutral hydrogen column density measured is consistent with that in the Local Cloud of 1-2 $\times 10^{18} \mathrm{~cm}^{-2}$. To make the non-detection of EUV flux consistent with the $\mathrm{C}$ and $\mathrm{B}$ band soft $\mathrm{x}$-rays using slab absorption requires a neutral column a factor of 10 higher, placing the emission beyond the local void, which is also inconsistnet with the softer Be band results. Maybe the void (defined by a lack of $\mathrm{N}_{\mathrm{HI}}$ ) and the bubble (defined by the $\mathrm{x}$-ray emitting hot gas) are not the same thing.

The DXS results (Sanders, this volume) detect diffuse emission in the $40 \AA-80 \AA$ range. However, no equilibrium models with or without elemental depletion fit the data well nor do non-equilibrum models tried to date. Our non-detection supports the view that solar abundance equilibrium models do not work, but also place a strong limit on the amount of $\mathrm{Fe}$ in the gas phase of a hot gas in equilibrium. A recent compilation of depletion measurements in neutral clouds towards many stars in the ISM indicate a minimum depletion of $\mathrm{Fe}$ of $0.5 \mathrm{dex}$, interpreted as either a compent of dust grains are indestructable in the cloud environment or that the abundance of $\mathrm{Fe}$ in the local ISM is not solar (Fitzpatrick, 1996). The lack of Fe in emission implied by our results $(0.78$ dex to make the EUV derived EM limits consistent with the B band limits) would suport either view. But the dust grains in this case are surviving a different environment of high $\mathrm{T}$, low density sputtering.

Our limits on the $\sim 200 \mathrm{~A}$ flux do not rule out all non-CIE models, and we are sure the B\&S overioninzed models can be made to be consistent by the manipulation of initial conditions. We used that particular model to demonstrate the usefulness of the EUV band to differentiate between CIE and nonCIE models. Not much more can be gleaned from the EUVE data set $18,000,000$ seconds is probably the longest astronomical integration in history! What is needed is an instrument with a large solid angle-effective area product combined with spectral resolution that can decouple the abundance and emission model effects that EUVE has done for stellar spectra. 


\section{References}

Jelinsky, P., Vallerga, J. and Edelstein, J.(1994): ApJ, 442, pp. 653-661

Fitzpatrick, E.L. (1996): ApJ, 473, L55-L58

Bratwurst, D. \& Schnitzel, T. (1994): Nature, 371, 774 


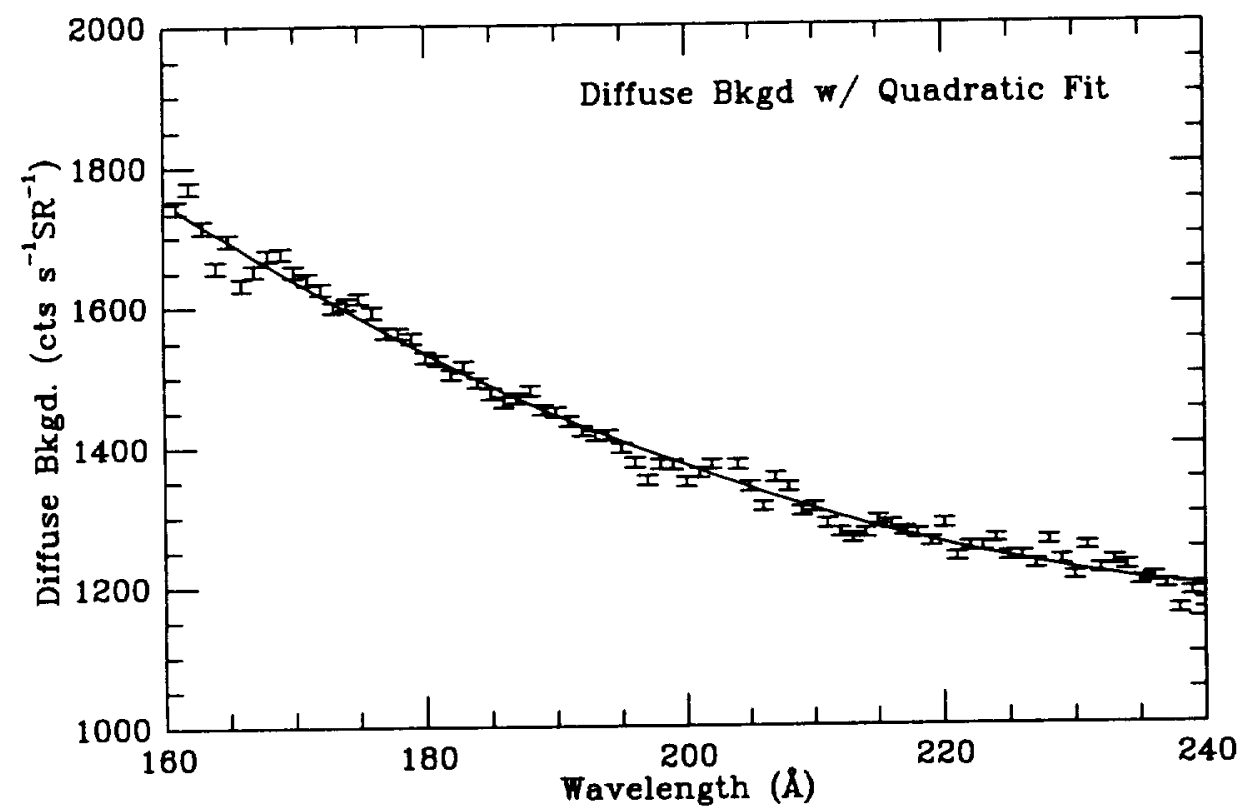

FIG 1 


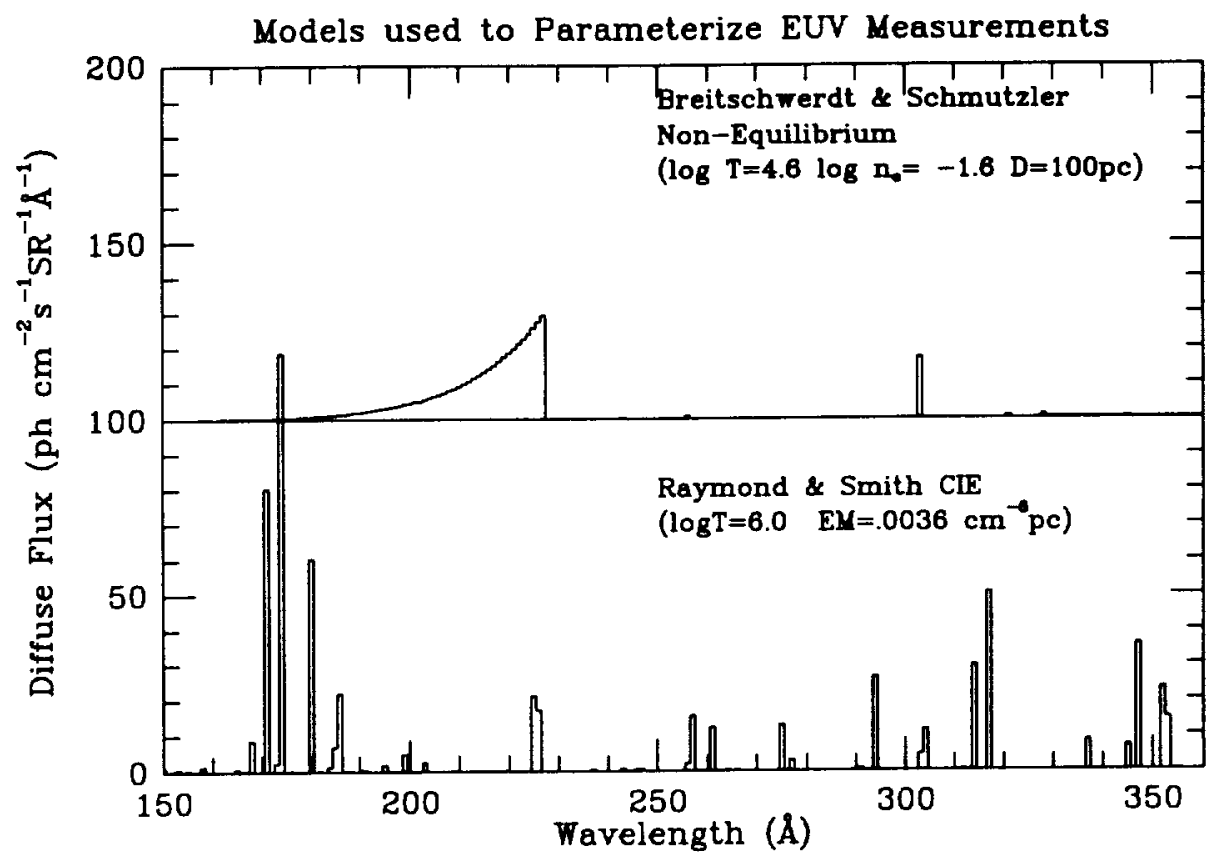

Fla 2 


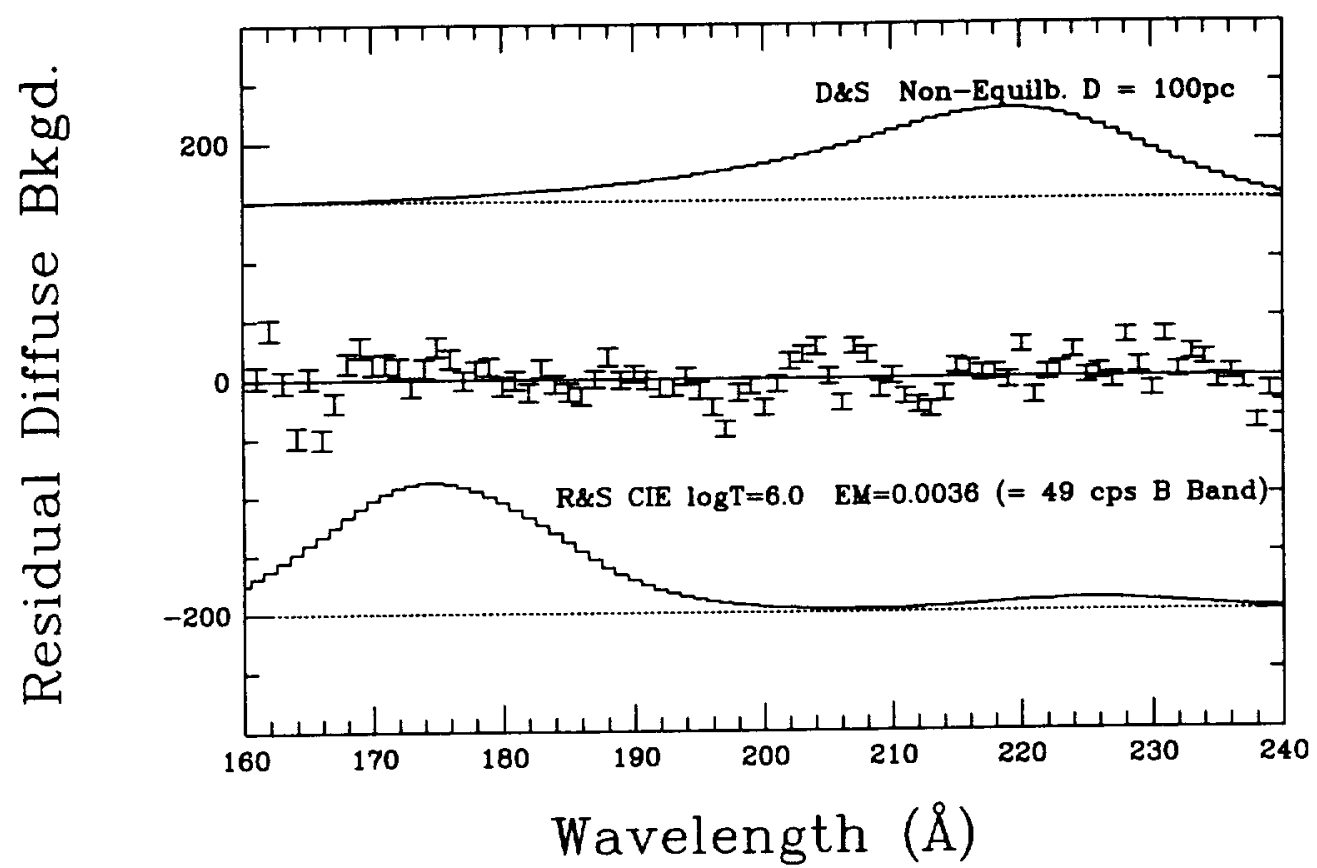

Fig 3 